\title{
Redes intelectuales y campo literario: Babel. Revista de Libros*
}

\author{
Intellectual networks and literary field: Babel. Revista de libros
}

Adriana Rodríguez-Alfonso**

\begin{abstract}
RESUMEN
El siguiente artículo analiza la red intelectual en torno a la publicación argentina Babel. Revista de Libros (1988-1991). Desde una perspectiva transdisciplinaria, se examina el entramado de sociabilidad, en diálogo con las estrategias de posicionamiento y legitimación de autores emergentes de la literatura latinoamericana del siglo XX, enfoque complementado con las humanidades digitales. Así, se revisará el aparato conceptual sobre redes intelectuales (Williams, Devés-Valdés, Maíz, Said), y luego se estudiarán los requisitos de estas relaciones ideo-estéticas e interpersonales, divididos en tres secciones: según el surgimiento, la formación y consolidación de la red.
\end{abstract}

\section{SUMMARY}

The following article analyzes the intellectual network of the Argentine publication Babel. Revista de Libros (1988-1991). From a transdisciplinary perspective, the framework of sociability is examined, in dialogue with the positioning and legitimization strategies of emerging authors of 20th century Latin American literature, an approach complemented by the digital humanities. Thus, the conceptual apparatus on intellectual networks will be reviewed (Williams, Devés-Valdés, Maíz, Said), and then the requirements of these ideo-aesthetic and interpersonal relationships will be studied, divided into three sections: according to emergence, formation and consolidation of the network.
Palabras clave: redes intelectuales, campo literario, Grupo Shanghai, revistas literarias, humanidades digitales.

Keywords: intellectual networks, literary field, Shanghai Group, literary magazines, digital humanities.

\footnotetext{
* Este artículo pertenece al proyecto doctoral en curso "Redes intelectuales y campo literario: el grupo Shanghai", auspiciado por una Beca Doctoral USAL-Santander en la Universidad de Salamanca.

** Cubana. Magíster, becaria predoctoral del Departamento de Literatura Española e Hispanoamericana de la Universidad de Salamanca. Personal Investigador en Formación del Instituto de Iberoamérica, y miembro del Grupo de Estética y Teoría de las Artes (GEsTA). Salamanca, España. a.rodalfonso@usal.es
} 


\section{Introducción}

¿Por qué Babel?

La misma conciencia, por tanto, debe tenerse de la existencia y del ser de su amigo, cosa que no es posible a no vivir con él cambiando en esta asociación palabras y pensamientos. Verdaderamente esto es lo que puede llamarse entre los hombres vida común.

ARISTÓTELES

El agitado campo cultural argentino de la década de los ochenta del pasado siglo fue un vívido reflejo de las fuertes transformaciones sociopolíticas en la dilatada transición hacia la democracia, tras el fin del régimen dictatorial, la elección de Raúl Alfonsín como presidente en diciembre de 1983, y la posterior gobernanza de Carlos Menem desde 1989 y sus correspondientes profundas transformaciones económicas y sociales. Los ochenta, decenio parteaguas a escala global, que conduciría con luces de neón al fin de cierto "siglo" y, tal vez, hasta de cierta "historia" del ajedrez geopolítico mundial', fueron el escenario de fondo de Babel. Revista de Libros (1988-1991), y del autodenominado "grupo Shanghai" que nuclearía a algunos de sus principales colaboradores, como Martín Caparrós, Sergio Chejfec, Alan Pauls, Daniel Guebel, Sergio Bizzio o Luis Chitarroni. El deslinde entre arte y política, el cuestionamiento de la función de la crítica, la defensa de una escritura separada de los imperativos de claridad, masividad y rentabilidad del mercado, signada en su lugar por la búsqueda de la experimentación formal (Noguerol Jiménez, "Narrar sin fronteras..." 29) - el significante que reemplaza en importancia al significado-, la autorreferencialidad y la creación de universos autónomos desligados de lo real, fueron algunos de los reiterados presupuestos que conformaron el ethos de la publicación. Programa este que, más que ser tomado ad litteram dadas sus contradicciones entre teoría y praxis, debe ser entendido como reflejo de su Zeitgeist y de

1 Aludo someramente a las teorías en torno al "siglo XX corto" de Eric Hobsbawm, comprendido entre el inicio de la Primera Guerra Mundial, y la caída de la Unión Soviética en 1991, así como a la controvertida tesis de Francis Fukuyama en The End of History and the Last Man (1992). 
los antagonismos, posicionamientos, y reestructuraciones del canon y la tradición de su campo cultural (Delgado 7).

Aunque fue una de las revistas más fundamentales del espacio literario porteño de esos años (Patiño 3), su efímera existencia, así como su escasa difusión fuera de Argentina, han hecho de Babel. Revista de Libros un pasaje casi "secreto" en la trayectoria escritural de los entonces jóvenes, parcial o totalmente inéditos ${ }^{2}$, que hicieron de esa publicación reducto propio en las luchas por la territorialidad entre las facciones intelectuales del campo, además de un vehículo para intervenir en la esfera pública y hacerse un lugar, paulatinamente, en ella. Precisamente, algunos años después del cierre de Babel. Revista de Libros, la presta carrera "ascendente" de buena parte de sus principales nodos como llamaré desde ya a las figuras centrales de este entramado ${ }^{3}-$ se hizo más que manifiesta: hacia mediados de los años noventa e inicios del 2000 se desplazaron con celeridad de las pequeñas e independientes editoriales nacionales que habían publicado sus primeros libros, a las importantes editoriales y consorcios españoles — antes criticados con asiduidad en las páginas del magazín-, así como de los iniciáticos reconocimientos locales, al sistema consagratorio de las conspicuas becas y galardones internacionales, con su consecuente resonancia en la crítica literaria y la academia.

Amén del probado valor estético de parte de las producciones individuales de los "babélicos", su originaria articulación colectiva, así como la probable prolongación de la red incluso después del fin de la revista ${ }^{4}$, deslizan las preguntas acerca de las particularidades de estas relaciones de sociabilidad, sus efectos en el posicionamiento y legi-

2 Por ese entonces solo unos pocos habían publicado sus primeros libros: Alan Pauls contaba solamente con la novela El pudor del pornógrafo (1984) y el ensayo Manuel Puig. La traición de Rita Hayworth (1986); Daniel Guebel con Arnulfo o los infortunios de un príncipe (1987); Caparrós, con Ansay o los infortunios de la gloria (1984) y No velas a tus muertos (1986); y Sergio Bizzio tenía publicado el poemario Gran salón con piano (1982). Mientras, Chitarroni y Chejfec aún eran inéditos.

3 En la teoría de redes los nodos son la serie definida de puntos o elementos referenciales entre los cuales confluye un conjunto de relaciones (líneas, vínculos o lazos) (Molina, J. 36).

4 La participación colectiva en antologías, libros de entrevista, homenajes, mesas literarias, presentaciones, así como las reseñas, y citas compartidas después del cierre de Babel. Revista de Libros en 1991 sugieren que los lazos entre sus principales integrantes se han mantenido. El estudio detallado de la prolongación de estos entramados en el tiempo forma parte del proyecto al que se adscribe este trabajo. 
timación en el espacio cultural, así como el posible influjo que tuvo la conformación de la "red" en el despegue de esos autores, más aún cuando esta génesis grupal ha sido notablemente omitida o desconocida por la recepción posterior de la obra individual de esos escritores. ¿Fue Shanghai un mero "grupo" de cordiales afinidades electivas? ¿O una red en toda su dimensión, con una estructura y distribución definida, una densidad de interconexión suficiente, espacios y medios autónomos, y un ethos compartido? Interrogantes estas que instan a restarle "informalidad" a la asociación de estos escritores, rebasando los límites del "mero grupo de amigos" pero que, fuera del territorio sociológico-descriptivo, exhortan también a leer la red como crisol colectivo tanto de sus obras como de sus curricula ulteriores, poniendo en crisis aquel hálito romántico o misticismo velado que pervive en la imagen de la creación aislada, la escritura ensimismada de los escritores en la soledad de sus bibliotecas, como diría Borges.

La demostración de la existencia e impacto de la "red" que se desarrolló y consolidó a través de los veintidós números de Babel. Revista de Libros en las páginas siguientes viene precedida de un sucinto recorrido conceptual, imprescindible para esta aproximación no muy visitada por los estudios del fenómeno literario. Posteriormente, el artículo analizará el origen, desarrollo y apogeo de la red, y para ello he divido en dos grupos los números de la revista señalando el número trece como parteaguas, cuando afloran los primeros créditos acopiados por el grupo, y también se inicia el sistema de reseñas, menciones y citaciones recíprocas que se desplegaría en abundancia hasta el cierre de Babel. A su vez, gracias a las herramientas digitales, el software libre Gephi me ha permitido representar no solo los distintos tipos de relaciones entablados entre los miembros de la red, amén de evaluarlos jerárquicamente, sino además visualizar los distintos estadios atravesados por el entramado, considerando la distribución y densidad de interconexión de esos lazos.

Finalmente, es necesario puntualizar que deliberadamente me he adscrito a la categoría "red intelectual" en detrimento de las emparentadas "red cultural" o "red literaria", ya que a pesar de la programática renuncia al "compromiso" del intelectual con lo real y el elogio de la literatura como universo autónomo postulado por los escritores de Shanghai - por las secciones de la revista, por el cariz de sus futuras 
producciones personales, y hasta por la intención misma tras el gesto de "publicar una revista", indicio claro del deseo de agitación, propaganda e intervención en la esfera pública (Sarlo, "Intelectuales y revistas" 7 ) - esos escritores están más cerca del intelectual "with a faculty for representing, embodying, articulating a message, a view, an attitude, philosophy or opinion to, as well as for, a public", proclamado por Edward Said (35), que de aquellos "escritores-cleros" de Julien Benda que, sumidos en la estratosfera del pensamiento, se guarecían de la tentación profana de contagiarse de las pasiones de la plaza pública (Benda 56).

\section{Para una historia de las redes intelectuales en Hispanoamérica}

Insertas en la encrucijada epistemológica entre la sociología, la historia de las ideas, la historia intelectual, los estudios culturales o las ciencias políticas, la categoría red intelectual ${ }^{5}$, cuyo origen deriva de la teoría de redes sociales, ha sido abordada desde diversas perspectivas teóricas durante las últimas décadas, encontrando especial fecundidad, en lo que al ámbito hispanoamericano se refiere, en las investigaciones referidas a la relevancia que tuvieron estos intercambios y entramados relacionales en la conformación de una hispanoamericana "república internacional del pensamiento" (Devés-Valdés 28), donde intelecto y práctica dialogaron estrechamente.

La travesía conceptual de los vínculos de la intelligentsia se remonta a la década de los ochenta del pasado siglo, cuando con el éxito de la teoría de grafos se formalizaron y delimitaron aquellas corrientes que enfatizaban la determinación de los vínculos relacionales en las consciencias colectivas - en detrimento de los atributos individuales como raza, sexo, o edad (Lozares Colina 118) - las cuales habían encontrado eco en las formaciones culturales de Raymond Williams ${ }^{6}$. Esta noción insta a interpretar las asociaciones entre artistas como algo

5 Algunos autores prefieren el concepto de "red cultural" (Fernández Bravo 23), pero dado el marcado carácter de intervención en el espacio público, el contenido de secciones como "Actualidad" o "Dossier", así como la vinculación posterior que escritores como Caparrós han mantenido con instituciones de activismo político social, he optado por el término "intelectual".

6 "Forms of organization and self-organization closer to cultural production" (Williams, Problems in... 57). 
mucho más complejo que el mero "grupo de amigos", centrándose en el ethos compartido, las expresiones de autopresentación y autodefinición (Williams, Problems in... 148), reaccionando así deliberadamente contra el ideal romántico que concibe las grandes obras y movimientos artísticos como resultado del artista genial, libre y aislado, e inclinándose, en cambio, por entender el surgimiento de un pensamiento original y coherente como el resultado de un esclarecimiento gradual y colectivo, que germina en las retroalimentaciones que resultan de esas asociaciones (Ringer 103).

En el campo cultural hispanoamericano, la estela de una sociología histórica del conocimiento puede rastrearse en nociones como "intercomunicación", propuesta por Ángel Rama -aunque este alude, más que a los vínculos entre sujetos, a los lazos supranacionales entre publicaciones periódicas en el continente (Rama 8) —; “religación”, ideada por Susana Zanetti y continuadora de la definición de Rama -si bien más explícita respecto de qué medios materializan esos vínculos, y de la relevancia de los presupuestos estéticos afines ${ }^{8}$ - o "coalición cultural" de Josefina Ludmer ${ }^{9}$, hasta arribar finalmente al puerto de la "red intelectual", enunciada por Eugenia Molina como el "conjunto de lazos que vinculan a los miembros de un mismo sistema social a través y más allá de las categorías sociales y los grupos cerrados" (Molina, E. 402). Con un fuerte trasfondo sociológico, Molina pondrá el acento sobre la funcionalidad de la red, la cual provee a sus miembros de "ayuda y asistencia" ${ }^{10}$, escenificando el "giro metodológico y temático" del en-

$7 \quad$ Otras propuestas que tributan a la teoría de redes en el plano cultural son las de Fernando Ortiz o, incluso, las de Mijaíl Bajtín, por solo mencionar algunas.

8 Zanetti menciona, por ejemplo, la correspondencia, las lecturas y encuentros colectivos, los "prólogos auspiciadores", así como ciertos emplazamientos o epicentros geográficos privilegiados para el intercambio (Zanetti 489).

9 La aproximación de Ludmer, de características muy concretas, se aleja de la línea dibujada por Rama y Zanetti, ya que estas asociaciones están intrínsecamente vinculadas con la institución estatal - siendo "ficciones para el Estado y con ellas se producen los sujetos del estado liberal" (Ludmer 48) -, subrayando el corpus de obras y las relaciones intertextuales que se establecen entre ellas, por encima de las interconexiones entre sus productores. Aún más, en un diálogo posterior, titulado "Encuentro con Josefina Ludmer", la pensadora argentina subrayará la especificidad de esta coalición cultural que ella restringe exclusivamente a la llamada Generación del 80 en Argentina (Dalmaroni 1123).

10 La red "reinserta al individuo en el marco de los grupos y las relaciones sociales en las cuales se mueve, y de las cuales asimila pautas de comportamiento, valores y creencias" (Molina, E. 399). 
foque que François-Xavier Guerra ya había empleado para la historia política.

Desde entonces, el devenir de los estudios relacionales sobre Hispanoamérica se ha reflejado en disímiles líneas, dentro de las cuales se localizan los trabajos de Marta Elena Casaús Arzú, Ricardo MelgarBao, Germán Alburquerque, Hugo Biagini, Eduardo Devés-Valdés, Eugenia Molina, Claudio Maíz, Álvaro Fernández Bravo, Margarita Merbilhaá, o César Zamorano Díaz, por solo mencionar algunos autores representativos. Amén de que algunos son abiertamente partidarios de los preceptos de la "historia de las ideas" (Devés-Valdés, Melgar-Bao, Biagini), mientras otros se adscriben al ala contraria, la de la "historia intelectual", que en el subcontinente ha tenido, entre otros, a Carlos Altamirano al frente de la palestra ${ }^{11}$, ambas tendencias parecen coincidir en las condiciones necesarias para definir una red, con independencia de si se privilegia una lectura "interna" o una "externa" (Dosse 180). Algunas de las posibilidades de esta visión ${ }^{12}$ son el enfoque colectivo y transnacional, la superación del concepto de "generación" (Maíz, "Las re(d)vistas..." 77) e "influencia" (Devés-Valdés 33), el acercamiento al Zeitgeist de una época, así como la explicación de los mecanismos de inserción institucional de los escritores principiantes (Merbilhaá 4), amén de las relaciones entre distintas propuestas individuales, y la ponderación de ciertas figuras respecto de otras.

Una zona mayoritaria de estos estudios apunta a leer la red como vía para la construcción de un pensamiento e identidad continental americana, ángulo especialmente privilegiado por los partidarios de la "historia de las ideas", donde las interconexiones entre intelectuales son vistas como clave para el desarrollo de la unidad intercontinental, como estrategia de salida del colonialismo y la marginación histórica, de ahí que el sentido último de corrientes como el arielismo, el humanismo teosófico, o el socialismo utópico, sea la fundación del territorio supranacional. Según esta perspectiva, el concepto "red intelectual" se

11 Entre los autores mencionados con anterioridad, Claudio Maíz, Fernández Bravo y Margarita Merbilhaá se han adscrito al camino prefigurado por Altamirano, explícitamente cuestionado, a su vez, por los feligreses del Corredor de las Ideas (Biagini 26).

12 Así mismo, existe un cúmulo de características relacionadas con la materialidad de la red, es decir, con las formas de comunicación específicas que la hacen posible. Estas serán comentadas en las páginas siguientes, ya que las pondré en praxis en el análisis del entramado intelectual nucleado en torno a Babel. 
opone al de "campo cultural", en cuanto la primera entraña cohesión, mientras el segundo conflicto ${ }^{13}$ (Devés-Valdés 35), visión que desestima la convivencia de ambos tipos de relación en el mismo espacio, cuando en el campo bourdieuriano la lucha de fuerzas para ocupar posiciones dominantes no suele ser un "agón" entre individuos aislados, sino precisamente, un enfrentamiento entre "bandos", dentro de los cuales se construyen, hermanados, densos y prolongados lazos. Es decir: redes. Como bien sostiene Bruno Latour: "Siempre es por comparar con los vínculos rivales que se destaca cualquier vínculo. De modo que por cada grupo a definir se establece también una lista de antigrupos" (54).

Otros autores, sin embargo, sí han considerado la relevancia de la red en los mecanismos de inserción de los actores implicados (Merbilhaá 4; Alburquerque 308), entroncándola con los mecanismos de adquisición de capital social y simbólico. Esta es precisamente la mirilla que más interesa en este trabajo: la red, articulada como celda de unidad de un grupo, pero también como meditada reacción frente a un otros que domina, y cuyo lugar se aspira a reemplazar; una estrategia que por fuerza desplegarán minuciosamente en sus páginas los principales colaboradores de Babel. Revista de Libros.

\section{Un Bildungsroman: Babel. Revista de Libros}

Génesis. Shanghai, un mito necesario

Shangai es, sobre todo, un mito innecesario.

MARTÍn CAPARRós (43)

La crisis, como mampara propicia para la red, ya ha sido sugerida por Raymond Williams ${ }^{14}$, y bien señala Roxana Patiño que Babel. Revista de Libros no fue el lugar de conformación del grupo Shanghai, sino el de su consolidación, pues desde inicios de la década de los ochenta sus miembros habían ido hilvanando vínculos (Patiño 4). Un recorrido por

13 "Por ejemplo, la noción ha contribuido a la propia creación de redes intelectuales y ello no es baladí, pues el concepto de 'campo', poniendo en relieve el conflicto, inhibe la creación de redes instalando la sospecha respecto de cualquier iniciativa (Devés-Valdés 35). 14 "Formations of the more modern kinds may be seen to occur, typically, at points of transition and intersection within a complex social history" (Williams, Sociology of Culture 134). 
esos años exhibe de forma larval algunas de las vías de comunicación imprescindibles para conformar una sólida red ${ }^{15}$, aunque la ausencia de otras que solo se concretarán con la fundación de la revista me impide designarla aún como tal.

Aunque con la revista este entramado incrementará sus miembros, la prehistoria de Babel. Revista de Libros evidencia ya las interconexiones entre su "núcleo duro"16. Se documentan encuentros "cara a cara" entre Daniel Guebel, Luis Chitarroni, Alan Pauls, Ricardo Ibarlucía, Daniel Samoilovich, Diego Bigongiari, Sergio Chejfec, Sergio Bizzio, Jorge Dorio y Martín Caparrós: primero, en la confitería Richmond, en la calle Florida, y luego, en La Ideal de Suipacha (Caparrós, "Mientras..." 526), espacio que será descrito en clave paródica años después en Los padres de Sherezade (2008), de Daniel Guebel, donde un personaje es invitado a "una confitería de moda", fundada bajo el montesquiano nombre de "L'sprit des Lois", pero luego llamada "La Ideal", y cuya pomposa descripción incluye "techos altos, pálidas velas de cera en candelabros de plata labrada, vitraux alegóricos, redondas y frías mesitas de mármol" (Guebel 31).

También participaron en proyectos comunes: Pauls y Guebel, en el efímero programa televisivo "Cinegrafía”, cuya continuación fue "Cable a tierra", en ATC (1984) (Figueras s/p), y Caparrós y Dorio, primero en "El sueño de Belgrano" (1984), y luego en "El monitor argentino", cuyas emisiones aparecen promocionadas en el cuarto y quinto número de Babel. Revista de Libros en 1988. Aunque aún sin medio colectivo, las publicaciones en Tiempo de Cultura, el suplemento de Tiempo Argentino (1982-1986), serán un espacio común, convirtiéndose en instancia privilegiada de construcción de una "identidad grupal"; es allí donde se gesta, junto con Página/12, el "nos" por escrito frente al que edificar un "otros", convirtiéndose en tarima para la autopresentación, las po-

15 Devés-Valdés sintetiza los requisitos indispensables para la existencia de una red: "encuentros cara a cara; correspondencia; la participación en los mismos congresos, sociedades, agrupaciones; la prologación, comentario o presentación de libros; la publicación en los mismos medios; la participación en las mismas campañas o iniciativas; los diálogos, polémicas; así como las citaciones recíprocas" (Devés-Valdés 32).

16 Sin pretender ser exhaustivos, en las páginas de Babel. Revista de Libros escribirían habitualmente Matilde Sánchez, Marcos Mayer, Daniel Link, Jorge Dorio, Marcelo Cohen, Charlie E. Feiling, Ricardo Ibarlucía, Christian Ferrer, América Cristófalo, Nicolás Casullo, Graciela Montaldo, María Moreno, Ricardo Forster, Horacio González, Guillermo Saavedra y Guillermo Schavelzon, además del "núcleo" ya mencionado con anterioridad. 
lémicas y artículos-pancartas, como en "Los nuevos" (Guebel y Bizzio 3); "Narrativa, el capítulo que se viene" (Chejfec 5), o la "Bibliografía anotada del impreciso futuro" (Balcarce 2), escrito bajo el pseudónimo colectivo Horacio Balcarce (Avellaneda 93).

La mesa redonda coordinada por Dorio en el Centro Cultural San Martín en 1984, "Narradores inéditos de ayer, de hoy y de siempre”, en la que intervinieron Chejfec, Chitarroni, Guebel y Caparrós señalará la participación conjunta en los mismos eventos (AfterNews s/p) ${ }^{17}$. Allí se reiterará ese "nosotros" que se articula en contraposición a escritores como Miguel Briante, Osvaldo Soriano, Guillermo Saccommano, Antonio Dal Massetto, o Tomás Eloy Martínez, practicantes de la estética "narrativista" (Klein 2), y representantes de una izquierda que aún defendía la obligatoriedad sesentista de la literatura a participar en la política y que, amparados por el gobierno de Alfonsín, integraban el establishment porteño. Puesta la mesa, este "almuerzo" era presidido por Ernesto Sabato, máximo paladín del enlace entre literatura y compromiso, quien será objeto de frecuentes burlas en las páginas de Babel. Revista de Libros.

Este conflicto -entre "viejos" y "nuevos", "formalistas" y "contenidistas", "entronizados" y "emergentes" - parece ser el principal detonante de la formalización del grupo, incluso por encima de las correspondencias estilísticas y de cánones literarios ${ }^{18}$. Shanghai —nombre deliberadamente "exotista" en rechazo a quienes los calificaban de "dandys" y "posmodernos" (Warley 39) - surge a partir del artículo "Ya no es lo mismo ser inédito que olvidarse" de Miguel Briante, escrito en junio de 1983 (Briante 8), que provocó una carta colectiva que nunca llegó a publicarse pero los animó a afianzarse en la seña colectiva: "Ya que nos atacan juntos, defendámonos juntos" (Caparrós, "Mientras Babel” 527). En consecuencia, en 1987 se publicará un manifiesto que

17 La significación que este "relato ontológico" tuvo para el reconocimiento posterior del grupo es tal, que se ha hecho ritual el relato de esos primeros "años de formación". De hecho, en una entrevista inicial solo se incluían en la mesa a Chejfec, Chitarroni y Guebel, pero en un texto posterior Caparrós apuntó que también él estaba allí, y que el título original de la mesa fue "Autores inéditos de hoy y de siempre".

18 Así lo confiesa el propio Caparrós: "Voy a hablar a partir de un nosotros dudoso y dubitativo. Que quizás, como todo nosotros, se construya más por exclusión de ellos que por afinidades propias" (Caparrós, "Nuevos avances y retrocesos de la nueva novela argentina en lo que va del mes de abril” 43). 
posteriormente Caparrós ampliará en "Nuevos avances y retrocesos de la nueva novela argentina en lo que va del mes de abril", publicado en el número 10 de Babel. Revista de Libros. Ese "manifiesto" expuesto allí se concentrará considerablemente más en la condena del "enemigo" - la "literatura Roger Rabitt" - que en la definición precisa de esta "nueva narrativa" encarnada por ellos, y cuyos ambivalentes postulados - "extrañamiento", "desconfianza de las grandes tramas", el fragmento, la digresión y la ruptura de la linealidad espacial y temporal, y de los géneros literarios (Caparrós, "Nuevos avances..." 45)— reproducirán contradictoriamente los principales rasgos de la denominada estética posmoderna "periférica" en boga a pesar del reiterado énfasis común en negarla (Noguerol Jiménez, "Últimas tendencias..." 168).

Lo anterior señala el talante à rebours de Shanghai respecto de la línea literaria dominante, pero tanto o más en relación con quienes figuraban como sus exponentes, y evidencia las motivaciones de una voz colectiva que surgió primero en el discurso que rodea a las obras que en las obras mismas, porque la producción literaria de los "babélicos" era casi inexistente hasta 1990. En el marco de una profunda crisis editorial, la ambigua "nueva narrativa" enunciada por Caparrós en 1989 queda explicada por la ausencia de un corpus real, cuyo vacío debía ser llenado con una identidad hipotética, que trazaba siempre en teoría, un incomprobable linaje dentro de la tradición nacional e internacional, amén de enfrentarse a sus némesis. El paratexto suplía al texto en un grupo que conseguía llegar a las publicaciones periódicas, pero escasamente a las editoriales, y cuyo apremiante deseo de visibilidad se expresaba en la fábula de una narrativa que era, parafraseando el artículo de Chejfec, todavía "un capítulo por venir". Su enunciación colectiva oscilaba entre una promesa de novedad y la adscripción a una genealogía legitimante ${ }^{19}$, pero su muestra era tan menor que no podía ser evaluada. Tampoco la red había sido completada: faltaba un medio de publicación común, pero ante todo, un circuito de referencias, menciones, reseñas y ponderaciones recíprocas que no podría existir hasta que el mito prometido se hiciera realidad.

19 Entre los autores del canon babélico se encontraban Osvaldo Lamborghini, Ricardo Piglia, Juan José Saer, César Aira, Arturo Carrera o Alberto Laiseca, por mencionar solo algunos. 


\section{Los primeros doce números}

El primer número de Babel. Revista de Libros salió a la luz en abril de 1988. Con independencia de los presumibles encuentros entre los miembros del comité editorial ${ }^{20}$, el magazín acentuó desde el principio los contactos que se tejían fuera de sus páginas. De hecho, la sección "Sucesos argentinos", encargada de difundir las actividades culturales locales más relevantes, se concentró en publicitar los proyectos colectivos e individuales de sus colaboradores habituales, subrayando sus asociaciones y delimitando sus espacios propios (Maíz, "Tramas culturales..." 25) como el Centro Cultural General San Martín, o la Facultad de Filosofía y Letras de la Universidad de Buenos Aires (UBA) ${ }^{21}$, mapa de interconexión que combina los lugares de reunión característicos de la primera mitad del siglo XX - las cafeterías, los centros culturales- con la universidad, espacio propio de la segunda mitad (Altamirano 13). "Sucesos argentinos" opera, además, por omisión, pues los eventos ajenos a los miembros del grupo son escasos, de forma que desde las primeras páginas la publicación ejemplifica la doble topología intrínseca a las revistas que Beatriz Sarlo ha señalado: el espacio intelectual concreto por el que circulan, y el espacio imaginario donde aspiran a ubicarse idealmente (Sarlo, "Intelectuales y revistas" 12), ya que allí el campo porteño lucía como una "casa tomada" por los colaboradores del magazín, haciéndolos pasar como figuras dominantes a diferencia de lo que ocurría realmente en el campo.

Además de los encuentros informales, las mesas y actividades compartidas, durante los diez primeros números se perfila otra modalidad de asociación: la participación en publicaciones periódicas comunes divergentes a Babel. Revista de Libros, aunque a la vez intrínsecamen-

20 Durante los veintidós números los directores fueron Martín Caparrós y Jorge Dorio. Guillermo Saavedra fungió como jefe de redacción hasta el número 19, cargo asumido por Christian Ferrer durante los últimos tres números. Además, fueron encargados de secciones fijas Chitarroni ("Siluetas"), Sergio Chejfec ("Historias de vida"), Daniel Chirom - a quien le correspondió "Poesía/Teatro", y fue sustituido por C.E. Feiling a partir del número 6 hasta su partida a Reino Unido-, Horacio González ("Actualidad"), y María Moreno ("La mujer pública"), por solo mencionar algunos nombres.

21 Se promocionan cursos y talleres impartidos en el Centro por Graciela Montaldo, Luis Chitarroni, Arturo Carrera y Martín Caparrós en el número 4. En el número 12 se anuncia que Chitarroni y Marcos Mayer organizan un Taller de Crítica, mientras que en la entrega 18 se promociona un taller orquestado por Chitarroni y Guebel. Por su parte, en la UBA se celebran las jornadas que dan lugar al Dossier sobre Sarmiento en el número 5, o bien el ciclo "Narradores de hoy" difundido en el número 12. 
te vinculadas con ella: los diarios El Porteño (1982-1993) y Página/12 (1987-actualidad), y la revista Diario de Poesía. Información, creación y ensayo (1986-2012), que instauró un gesto rupturista semejante al babélico pero en el plano poético (Mattoni 48). En menor medida, también Último Reino (1979-1988), El Murciélago (1990-2005), Espacios (1984-actualidad), Farenheit 450 (1986-1988) o Tramas literarias (1989), se fueron dibujando como pares o extensiones de una red que responde más al mencionado término de "intercomunicación" de Rama, ya que se construye un entramado suprapersonal que rebasa los vínculos entre individuos aislados, y se anuda entre las publicaciones periódicas que los aglutinan ${ }^{22}$. La materialidad de estos vínculos se traza, en primera instancia, a través del circuito publicitario desplegado en Babel. Revista de Libros, donde son anunciadas con mayor o menor frecuencia - como El Porteño, que será promocionado solo en los primeros cinco números, mientras que Página/12 aparecerá ritualmente en las veintidós ediciones, llegando a ocupar, simbólicamente, la propia "página 12" del magazín-; así como las correspondientes secciones de reseñas de poesía y teatro, psicoanálisis, o ensayo, donde sus números son comentados y recomendados. Además, a menudo los comités editoriales de esas publicaciones estaban integrados por asiduos colaboradores de Babel, como David Samoilovich, Ricardo Ibarlucía, Jorge Fondebrider (Diario de Poesía), Germán L. García (El Murciélago) o Christian Ferrer (Farenheit 450), por solo mencionar algunos, o bien pertenecían a su circuito editorial, como sucede con Último Reino, en donde se publican poemarios de los babélicos Guillermo Saavedra o Jorge Dorio, o de los autores "canonizados" por Babel, como Carrera o Perlongher, y cuya labor de traducción y divulgación de la poesía latinoamericana es ensalzada, en contraposición al hispanófilo mercado peninsular (Ricagno 44). Otras editoriales independientes que cumplen esta doble función, siendo objeto de promoción y a la vez medios de publicación de la red, son Ada Korn Editores y Ediciones de la Flor, cuyos responsables tendrán "tarima" en la sección "Tráfico.

22 Esta red será, además, supranacional, pues un número considerable de sus colaboradores principales terminarán escribiendo desde Brasil, Venezuela, España, Reino Unido o Austria. 
Una tribuna para mercaderes" (1-14), en ocasiones también evidente valla autopublicitaria ${ }^{23}$.

Junto con algunas de las ideas y a comentadas en el primer apartado del trabajo algunos de los elementos que conformaron los leitmotiv de la poética colectiva de la revista - su ethos-son:

- la apuesta de riesgo por propuestas noveles y transgresoras frente a las voces de siempre, de ventas seguras y dominantes en el "ranking del mes";

- la conjunción de la literatura con actualizadas aproximaciones provenientes de la filosofía, las ciencias sociales y políticas, y la psicología;

- el llamamiento a una apertura - cultural, epistemológica-a otras latitudes que redujera el provincianismo nacional e hiciera al campo porteño "contemporáneo al mundo", a la par que se clamaba por la subvención estatal para sobrevivir a los consorcios, y se hacía una elegía de la perdida centralidad de los años dorados del sistema editorial argentino, usurpado ahora por el zaherido mercado editorial español.

Estas ideas eran frecuentemente compartidas por las publicaciones y editoriales colindantes, aportando así un elemento más para situar a sus cófrades en el perímetro extensivo de la "familia" Babel. Además, al circuito editorial y medial circundante se sumaba la institución universitaria, cuyo canon literario solía interceptarse con el babélico, ya que muchos de los colaboradores de la revista estaban asociados al ámbito institucional. Aún más, este entramado entrañaba un fuerte efecto legitimante, tanto de la revista, como de sus noveles escribientes, ya sea de manos de académicos con un creciente grado de reconocimiento,

23 Babel tiene aquí uno de sus espacios de intercambio de capital simbólico, ya que los editores que hablan en sus páginas comentan la crisis editorial nacional, a la par que se autoproclaman los paladines sobrevivientes en la lucha entre las cuidadas pequeñas editoriales argentinas, y las pecuniarias y de dudoso juicio estético grandes editoriales españolas, símbolos del mercado neoliberal. Mientras, los babélicos reciben también sus respectivos créditos, ya que a menudo los editores aprovechan para recomendar algunos de sus libros, entre los que no faltan los de los babélicos: en el número tres, por ejemplo, Daniel Divinsky, de Ediciones de la Flor, señala su apoyo a los "noveles y novelas" (Divinsky 6). Más explícito, el editor de Ada Korn, Salvador Pazos, apunta que "los dos primeros títulos de ese proyecto bastante personal, necesariamente modesto, casi artesanal, de producción de libros fueron Ansay o los infortunios de la gloria, de Martín Caparrós y El vestido rosa. Las ovejas, de César Aira" (Pazos 6). 
como Graciela Montaldo o María Teresa Gramuglio, o bien, a través de otros ya consagrados, como Noé Jitrik o Beatriz Sarlo, llegando esta última a dedicar su "mesa de luz" al inédito El Coloquio, de Alan Pauls (Sarlo, "La mesa de luz" 20). Esta práctica, nacida en los folios del medio, se prolongaría en las décadas siguientes, tal y como ilustra la propia Sarlo en el caso de Chejfec (Rodríguez Alfonso 2).

Esos medios y figuras compartían postulados ideo-estéticos con $\mathrm{Ba}$ bel. Revista de Libros, y articulaban así un sistema de replicación y refracción donde el programa babélico y el nuevo canon literario que le servía de fundamento se reproducían como eco que se replica y expande ${ }^{24}$. Por otra parte, esta "onda" era recíproca, ya que miembros del comité editorial de Babel se vinculaban también al El Porteño, como Caparrós, tal y como cuenta María Moreno en una crónica suya (Moreno 1), y, sobre todo, a Página/12, a cuyo comité pertenecieron Dorio y el mismo Caparós, y en donde también Pauls escribiría con frecuencia.

Esto último conduce a otro elemento conjuntivo: la intervención en polémicas y diálogos que delinean bandos en pugna, y acentúan, a su vez, la densidad de vínculos entre los miembros de la red dado el carácter "relacional" de los agentes que intervienen en el campo (Bourdieu 271-272). En este sentido, es necesario hacer una salvedad respecto de El Porteño y Página/12, pues a pesar de que los babélicos escribían allí, su dirección estaba integrada por Briante o Soriano, ambos mencionados representantes del "bando opositor" delineado por los jóvenes, lo que explica porqué, a pesar de su aparente alianza, Dorio y Caparrós fueran expulsados de Página/12 en 1990 por Soriano ${ }^{25}$. De hecho, el performance de los grupos en pugna se haría evidente en secciones como "Vanidades", que posteriormente sería sustituida por "Ripios argentinos" de Marcos Mayer, y cuya función era exclusivamente lanzar críticas y burlas a Miguel Briante, Osvaldo Soriano, Tomás Eloy Martínez, Oscar Steimberg, Félix Luna, y otros tantos más representan-

24 Por su relevancia, los vínculos entre las operaciones de canon y la red intelectual desplegada en la publicación periódica han sido abordadas en otro artículo en prensa titulado "Instrucciones para implosionar un canon. Babel. Revista de Libros".

25 Para un relato más detallado del conflicto entre Briante, Soriano y los babélicos en 1990 véase (Castro 3). 
tes del establishment cultural porteño, a pesar de la evidente dosis de impostura que siempre implican estos antagonismos ${ }^{26}$.

Sin embargo, otro "antigrupo", mucho más interesante, era delineado con bastante más sutileza en las secciones de reseñas, especialmente las de "Narrativas", donde con frecuencia eran destrozados -aunque siempre bajo argumentos estéticos y/o ideológicos-, los libros de jóvenes galardonados con reconocimientos literarios que no pertenecían al grupo babélico, críticas con frecuencia generadoras de réplicas y polémicas. Amilia Jamilis, quien había ganado el Premio Fondo Nacional de las Artes 1988 (6); Sylvia Iparraguirre, ganadora del Primer Premio Municipal de Literatura (6); Lisa Peluffo, ganadora del Premio Emecé 88/89 (12), así como los primeros libros de Carlos Ares y Enrique Zatarra (9), son algunos de los disímiles coetáneos que recibieron la ofensiva babélica, y con los cuales, al menos en las páginas de la revista, el "agón" parece ser más explícito que con los "planetarios" o miembros de Con V de Vian a quienes se suele contraponerlos ${ }^{27}$. Todos estos elementos, en definitiva, delineaban una red relativamente policéntrica hasta el número 12 (Ilustración 1 y 2), cuya estructura interna parecía ser bastante equilibrada, ya que ninguna figura particular resaltaba o reunía un número relevante de interconexiones. Apenas existían lazos bidireccionales explícitos entre los integrantes del grupo, es decir, más allá de alguna mención o comentario aislado, solo la publicación en un medio común evidenciaba las conexiones concretas existentes entre ellos, elemento de por sí poco sustancial, dado que se analizan precisamente los lazos dentro de esa publicación. Esta distribución, sin embargo, cambiaría notablemente en las siguientes entregas de la mano de un incremento de capital simbólico.

26 Es evidente, por supuesto, que no puede olvidarse el cariz de impostura, o postura autorial implícito en toda polémica, de la cual, además, la historia literaria argentina es rica, desde el enfrentamiento entre Florida y Boedo, hasta la confrontación entre las revistas Sur y Contorno.

27 El también "mítico" enfrentamiento entre "babélicos" y "planetarios" ha sido puesto en duda en más de una ocasión, y aunque es recurrente mantenerlo (Cohen s/p), todo parece indicar que el enfrentamiento pertenecía más al orden del "discurso" que al de la "realidad". De hecho, tanto Juan Forn como Rodrigo Fresán compartieron mesa con los Shanghai e, incluso, escribieron de forma ocasional en Babel. Revista de Libros: el primero en la crediticia sección "La mesa de luz" en el número 21, y el segundo en la también relevante "El libro del mes", a propósito de Fogwill en el numen 20. 

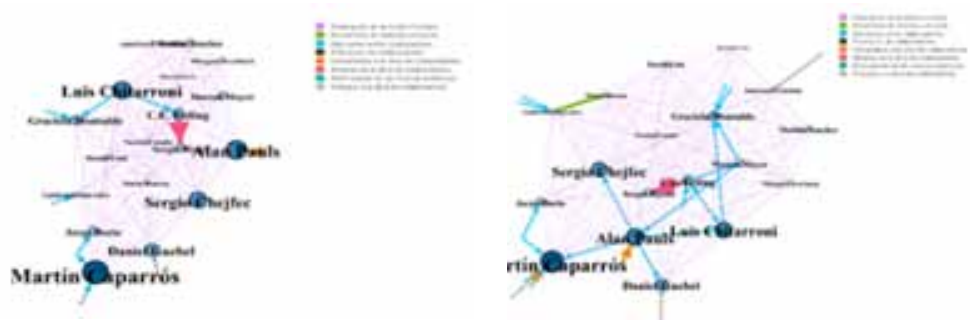

Ilustración 1. Distribución de la red Ilustración 2. Distribución de la red en el número 4 de Babel. Revista de en el número 10 de Babel. Revista de Libros. Libros.

Conquistar el bronce: los números 13-22

Príncipe poderoso soy, a pesar de mi origen oscuro.

HORACIO (81)

A partir del decimotercer número, la voluntad aperturista de la publicación se acentúa, y secciones como "Lecturas" participarán de forma más activa en la divulgación y traducción de la literatura mundial, sumándose al carácter cosmopolita de los monográficos de "Bárbaros", o las compilaciones del "Dossier". En cuanto a la estructura interna, al tiempo que muchas de las secciones originales desaparecen, esta se diversifica en otras de frecuencia fija u ocasional, como "Batidore Liberto", "El cónsul honorario", “Ripios nacionales", “La Verónica” o "La biblioteca del mes", generalmente asociadas con los nodos de mayor peso en la red. Al mismo tiempo, Babel se "expande" a otros formatos, y aparece desde las nueve de la noche hasta las cuatro de la mañana en el 97.5 FM de Radio Clásica ${ }^{28}$. Cuestión que marca una diferencia en relación con el "El monitor argentino", ya que este se afiliaba a la red solo a través de sus directores comunes, mientras que el programa radial se instaurará como explícita extensión de Babel, y dará cuenta de la relevancia que había adquirido la publicación en el campo cultural porteño para ese entonces ${ }^{29}$.

28 Este programa será publicitado en el magazín de los números 9 al 15.

29 "El monitor argentino" fue un programa televisivo de divulgación literaria dirigido por Caparrós y Dorio, los también directores de Babel. De efímera existencia, sus capítulos fueron promocionados en los primeros números de la revista. 
Pero la transformación más sustancial pertenece al orden que aquí interesa: la encrucijada entre la red intelectual tejida en torno a $\mathrm{Ba}$ bel. Revista de Libros y el paulatino posicionamiento de sus principales nodos en el campo. Esto ocurrirá a partir del número 13, cuando Daniel Guebel gana el Premio Emecé 89/90 con La perla del emperador, lo que da pie a que se publique un fragmento suyo en una de las emblemáticas secciones de autorrepresentación grupal, "Caprichos", titulada "Lars Vigdom". Esto marcará the turn of the screw de la asociación, pues la figura autorial, el rol de los babélicos, tornará de "críticos" a "escritores", cosa que en paralelo propiciará la condición faltante para confirmarlos como red intelectual: el circuito de citas, reseñas, menciones y prólogos mutuos.

La legitimación y visibilidad del galardón de Guebel, sumado a la inminencia de publicación colectiva, espoleará vertiginosamente la forma in crescendo en que los nodos van "copando" las secciones de la revista: en el número 14 el "anticipo" promocionado será El oído absoluto de Marcelo Cohen, y en el 15 se coloca el poemario "La mujer pez" de Dorio, prologado por María Moreno. También se incluye un cuento de Chitarroni, especie de Gertrude Stein talismánico del grupo, en el apartado "Lecturas", destinado mayoritariamente a la traducción y divulgación de textos poco conocidos de autores fundamentales de la tradición occidental, como Mark Twain, Rainer María Rilke, Henri Michaux o Ezra Pound. Así mismo, a partir del 14 se promocionará durante seis números consecutivos "El tercer cuerpo" de Martín Caparrós, superando todos los rankings de promociones de un mismo libro que hasta entonces habían existido en la revista.

En el número 16 suceden dos hitos definitivos: aparecen juntos en la mesa "Nueva narrativa argentina; alcances y posibilidades" en la Feria del Libro ${ }^{30}$, y reciben la beca de la Fundación Antorchas Sergio Bizzio (El divino convertible), Martín Caparros (La noche anterior), Sergio Chejfec (Moral) y Alan Pauls (El coloquio). De forma bidireccional, estos hechos indican, por un lado, el capital simbólico que Babel ha adquirido a dos años de su surgimiento y, en parte gracias a

30 Publicitado en "Sucesos argentinos", los invitados fueron Sergio Bizzio, Martín Caparrós, Sergio Chejfec, Jorge Dorio, Juan Forn, Daniel Guebel, Graciela Montaldo, Alan Pauls y Matilde Sánchez, y contó con la coordinación de Luis Chitarroni. 
ella, sus nodos más importantes, llevándolos a tener un espacio propio de resonancia internacional como es la Feria del Libro; pero, al mismo tiempo, estos reconocimientos, anunciados a toda página en "Sucesos argentinos", legalizan a ojos de su público la estrategia $a u$ topromocional que se desplegará en los próximos números. Pues resulta que los textos elegidos para "El libro del mes" del número 17 al 19 serán sucesivamente Lenta biografía, de Sergio Chejfec, La perla del emperador, de Daniel Guebel, y El coloquio, de Alan Pauls, en una sección hasta entonces ocupada por autores de prestigio mundial, como Thomas Bernhard o Umberto Eco, o bien, por representantes del canon nacional alternativo propuesto por Babel, como Juan José Saer, Witold Gombrowicz u Osvaldo Lamborghini. Es decir, aparecerá en la sección de mayor jerarquía de la revista el segundo libro de Guebel y la ópera prima de Chejfec, en un giro que, sin dejar de ser súbito, tampoco asombrará demasiado puesto que se ha ido fabricando gradualmente.

En cuanto a la red, al abandonar su condición cuasi inédita los principales nodos finalmente obtienen su enlazamiento formal (Ilustración 3). Sin querer ser exhaustivos, en el número 17 Pauls reseña la novela de Chejfec, y se reproduce un fragmento del prólogo preparado por C. E. Feiling; luego, Daniel Link y nuevamente Pauls rinden homenaje a Guebel en el número siguiente, y también Caparrós le dedica más adelante un elogioso comentario en "La Verónica" (19). Por su parte, Guebel aprovechará para mencionar a Chitarroni en su "autocomentario" sobre La perla del emperador, quien a su vez reseña junto con Alejandro Katz El coloquio de Pauls en el número 19. A partir de entonces, y a falta de libros, las menciones fortuitas se multiplican y se alude a la obra de Guebel y Pauls en un texto sobre $L a$ aventura semiológica de Barthes (20), o se subraya que se "aprecian las lentas biografías", clara alusión a la novela de Chejfec, a raíz de una semblanza de Sartre (21), amén de los relatos que en esa misma entrega C. E. Feiling, Caparrós y Chitarroni hacen pasar por "ensayos" en el Dossier nada más y nada menos que dedicado al tango. Este circuito de citaciones y menciones recíprocas transformará radicalmente la composición de la red, poniendo en un primer plano a ciertas figuras por encima de otras (Ilustración 4). 


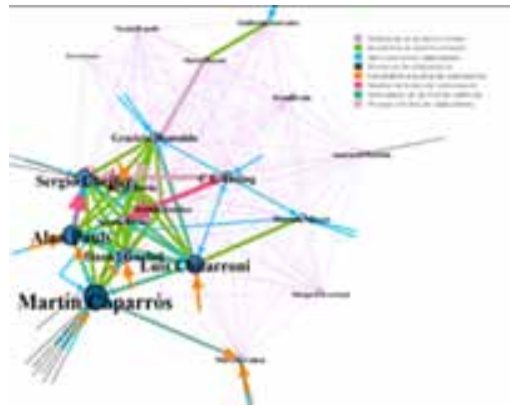

Ilustración 3. Distribución de la red en el número 17 de Babel. Revista de Libros.

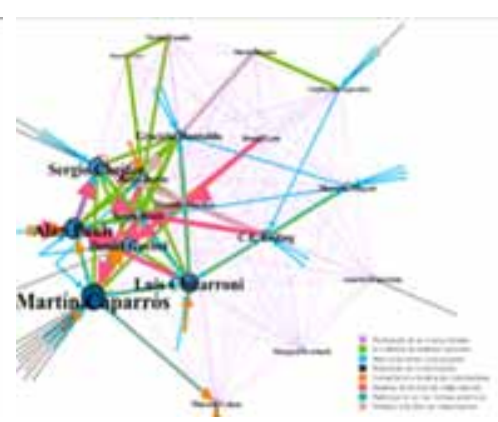

Ilustración 4. Distribución de la red en el número 22 de Babel. Revista de Libros.

Amén de las polémicas en las que también se involucran de forma colectiva durante estos números - como la ya relatada con Briante y Soriano en Página/12, o las relacionadas con Lamborghini y Aira en la misma Babel-, la introducción de las obras de los babélicos, así como el circuito crítico orquestado para su justificación, no solo formalizará la red, sino que definirán su estructura definitiva. Realmente, hasta el número 13 las menciones, comentarios y citas colectivas son bien escasas, de ahí que el tipo de relación predominante sean las publicaciones en el mismo medio, cuestión que haría pensar que figuras con colaboraciones frecuentes como Marcos Mayer, Marcelo Cohen, Graciela Montaldo o Daniel Link tienen más peso en la red que los jóvenes escritores que en 1987 habían firmado el "manifiesto" Shanghai. Sin embargo, a partir del "logrolling" o quid pro quo desplegado por el premio de Guebel (Harris 53), la distribución interna del grupo se transformará radicalmente, y en los números siguientes se clarificará quiénes son los miembros con el mayor número de interacciones de esa red policéntrica (Ilustración 5).

Con excepción de Chitarroni, quien escribe número tras número la columna "Siluetas", las colaboraciones de Caparrós, Chejfec, Pauls y Guebel son considerablemente menores en número que otros participantes de Babel. No obstante, ninguna de esas otras figuras recibirá la "atención" - cifrada en el conjunto de citas, reseñas, prólogos, entre otras formas de interrelación respecto de las que ya he insistido- de las cinco figuras que se perfilan "centrales" del entramado. Así mismo, al haber analizado la conformación de la red en un único medio, he ponderado más estas otras formas de comunicación, considerando 
que la coexistencia de textos en un mismo número será algo relativamente común, mientras que la referencia particular a ciertas figuras, las cuales resultan "distinguidas" en el sentido bourdieuriano, resulta considerablemente más significativa.

En términos de red, Chitarroni, Caparrós, Chejfec, Pauls y Guebel son los nodos con mayor densidad de lazos (Ilustración 6), pero en cuanto al campo intelectual, son también esos autores quienes acopian, como bien señala Merbilhaá, el mayor capital social y simbólico, instituyéndose como "líderes de opinión" y "consagradores" (Merbilhaá 5). Esto último queda claro a partir del número 17, cuando adoptan indistintamente los roles de "creadores" o "críticos" en función de las circunstancias, legitimando con sus aplausos la incipiente obra de sus pares, y recibiendo, simultáneamente, los créditos que estos les otorgan. Cuestión que no hubiera sido posible sin la existencia de un proyecto común: Babel. Revista de Libros, que se erguirá como instancia aglutinante de la red, pero, a la vez como consecuencia de esta.

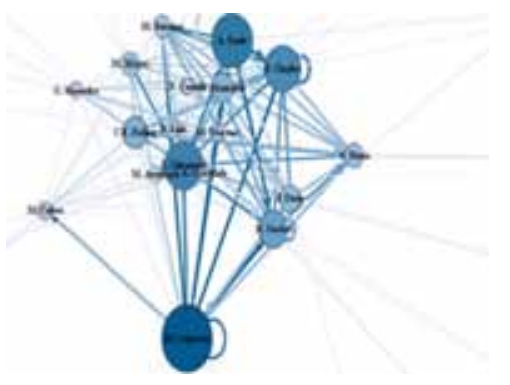

Ilustración 5. Detalle de los nodos Ilustración 6. Densidad y peso de los

principales de la red en el número 22 nodos principales de la red. de Babel. Revista de Libros.

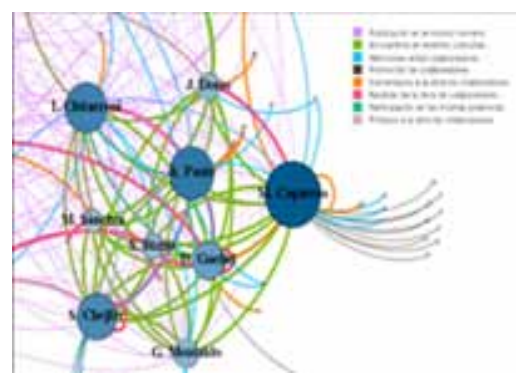

Cuando en marzo de 1991 la publicación dejó de existir, los núcleos de la red resultante coincidían en casi su totalidad con los escritores poco "éditos" tras el mito Shanghai de principios de los ochenta, como puede observarse en la Ilustración $7^{31}$.

31 Algunas excepciones de esta afirmación son los casos de Sergio Bizzio o María Moreno. Décadas después, Bizzio es, efectivamente, otra de las figuras más reconocidas de esa generación, y tal y como revisé en el primer apartado sobre Babel, él fue uno de los integrantes del Grupo Shanghai; sin embargo, su participación en la revista fue prácticamente nula, publicando solamente una reseña sobre Jack Higgins en el número 1. Es cierto que se le menciona en más de un encuentro colectivo, y que tanto Mínimo 

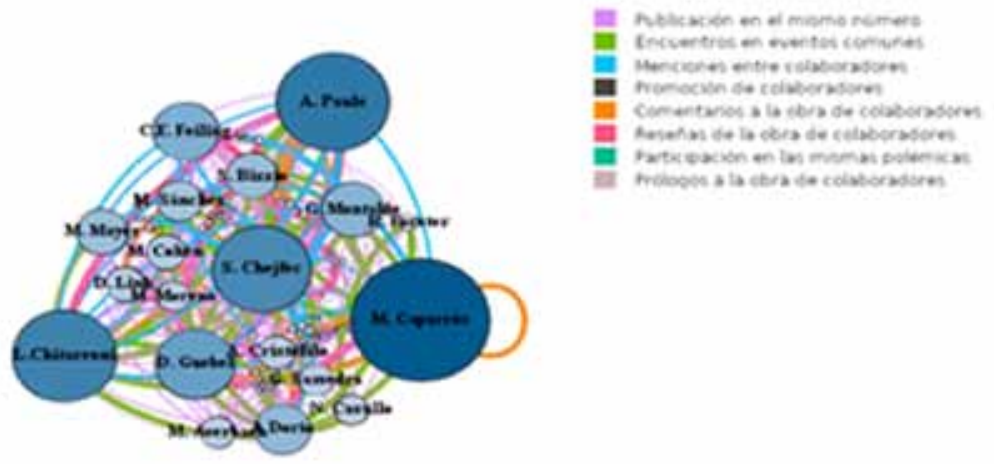

Ilustración 7. Intensidad y dirección de las relaciones que confluyen hacia los principales nodos.

Al mismo tiempo, a principios de los años 2000, eran también esas figuras quienes ostentaban el mayor acopio de capital simbólico, lo que ratifica nuevamente el parentesco entre los entramados de sociabilidad y las luchas por el posicionamiento, así como la representatividad de los escritores emergentes, para quienes el convivir (syzên) fue no solo una alianza de afinidades, sino una plataforma de inserción futura ${ }^{32}$.

\section{Conclusiones}

En las páginas precedentes me propuse analizar el entramado de relaciones fundado alrededor del medio Babel. Revista de Libros a fines de la década de los ochenta en Argentina, y el impacto que las alianzas allí tejidas tuvieron en la representatividad e inserción ulterior de sus principales figuras. Luego de un sucinto recorrido por el abordaje conceptual de las "redes intelectuales", el artículo se adentra en las condiciones necesarias para conformar una "red", para posteriormente demostrar que las relaciones entabladas entre los colaboradores de la revista rebasaron con creces el nicho informal del "grupo de amigos", viabilizando el gradual reconocimiento de estos escritores emergentes.

figurado como El divino convertible fueron reseñados en Babel, pero aparece siempre como una figura ausente que recibe los créditos del ritual consagratorio, pero que no participa de ello. María Moreno, por su parte, escribe durante los primeros números de forma regular en su propia columna, pero su intervención en la revista se restringe casi exclusivamente a esas colaboraciones.

32 Para Giorgio Agamben, es esta "convivencia", esta participación de una comunidad colectiva, la clave de las teorías de Aristóteles sobre la amistad (Agamben 5). 
Puesto que el enfoque privilegiado ha sido el cruce entre la teoría de redes intelectuales y los procedimientos de posicionamiento en el sistema literario, el trabajo ha combinado continuamente ambas perspectivas de análisis, cuya ejemplificación, además, se ha representado a través de un conjunto de visualizaciones con la intención de hacer confluir, las herramientas brindadas por las humanidades digitales.

Debido al considerable número de datos trabajados en este artículo - las múltiples interconexiones dadas entre los miembros de la red a lo largo de los veintidós números de Babel. Revista de Libros, cuyas entregas solían superar las cincuenta páginas-, la plataforma Gephi ha permitido procesar la base de datos construida, ofreciendo, la posibilidad de identificar patrones y tendencias, aislar ciertos nodos, o segmentar etapas del proceso de religación y consagración articulado doblemente en el magazín. Los resultados de este nuevo camino de ninguna forma reemplazan las aproximaciones habituales, sino que sirven de complemento a la metodología teórica y exegética tradicional.

\section{Referencias bibliográficas}

AfterNews. "A 20 años del cierre de la revista sobre libros Babel". Notife, 19 de junio de 2011. Recuperado de https://notife. com/320616-a-20-anos-del-cierre-de-la-revista-sobre-libros-babel/

Agamben, Giorgio. “La amistad”. La Nación, 25 de septiembre de 2005. Recuperado de https://www.lanacion.com.ar/cultura/laamistad-nid741397/

Alburquerque Fuschini, Germán. "El caso Padilla y las redes de escritores latinoamericanos". Revista Universum, vol. 16, 2001, pp. 307-320.

Altamirano, Carlos (Dir.). Historia intelectual en América Latina, vol. I \& II. Buenos Aires, Madrid, Katz Editores, 2010. Impreso.

Avellaneda, Andrés. "Bioy, pasado y presente". Espacios de crítica y producción, vol. 32, 2005, pp. 92-101.

Balcarce, Horacio. "Bibliografía anotada del impreciso futuro". Tiempo Argentino [Buenos Aires]. 6 de nov. 1983, p. 2.

Benda, Julien. La Trahison des clercs. Paris, Éditions Grasset, 1927. Impreso. 
Biagini, Hugo E. "La historia de las ideas, sus corredores y la historia intelectual." Cuadernos Americanos, vol. 2, no. 160, 2017, pp. 11-28.

Bourdieu, Pierre. Las reglas del arte: génesis y estructura del campo literario. Barcelona, Anagrama, 1995. Impreso.

Briante, Miguel. "Ya no es lo mismo ser inédito que olvidarse". Tiempo Argentino [Buenos Aires]. 4 de mayo 1984, p. 8.

Caparrós, Martín. "Mientras Babel". Cuadernos hispanoamericanos, vol. 517, 1993, pp. 525-528.

"Nuevos avances y retrocesos de la nueva novela argentina en lo que va del mes de abril". Babel. Revista de Libros, no. 10, 1989, pp. 43-46.

Castro, María Virginia. “¿Posmodernos? ¿Apolíticos? ¿Grupo Shangai?: nuevas narrativas sobre la última dictadura militar". VII Congreso Internacional Orbis Tertius de Teoría y Crítica Literaria, 19, mayo, 2009, La Plata, Centro de Estudios de Teoría y Crítica Literaria, Facultad de Humanidades y Ciencias de la Educación, pp. 23-34.

Chejfec, Sergio. "Narrativa, el capítulo que se viene". Tiempo Argentino [Buenos Aires]. 6 de nov. 1983, p. 3.

Cohen, Selma. "Nuevas coordenadas de la literatura argentina". La Voz [Buenos Aires], 4 de agosto de 2020, http://archivo.lavoz. com.ar/2002/0104/suplementos/cultura/nota75496_1.htm

Dalmaroni, Miguel. "Encuentro con Josefina Ludmer". Orbis Tertius, vol. 4, no. 7, 2000, pp. 1851-7811.

Delgado, Verónica. “Babel. Revista de Libros en los ‘80. Una relectura”. Orbis Tertius, vol. 1, no. 2, 1996, pp. 23-50.

Devés-Valdés, Eduardo. Redes intelectuales en América Latina. Santiago de Chile, Universidad de Santiago, 2007. Impreso.

Divinsky, Daniel. "Reivindicación de libros". Babel. Revista de Libros, no. 3, 1988, p. 6.

Dosse, François. "De la historia de las ideas a la historia intelectual". Historia y grafía, vol. 19, 2002, pp. 171-192.

Fernández Bravo, Álvaro. "Redes culturales del 80: alianzas, coaliciones y políticas de la amistad". Historia de la Literatura Argentina. El brote de los géneros. vol. III. Ed. Noé Jitrik, Buenos Aires, Emecé, 2010, pp. 385-412. Impreso. 
Figueras, Marcelo. "El Sub 65 pide pista". Página/12, 13 de noviembre de 2011, www.pagina12.com.ar/diario/elpais/1-181132-2011-11-13.html

Guebel, Daniel. Los padres de Sherezade. Buenos Aires, Eterna Cadencia, 2008. Impreso.

Guebel, Daniel y Sergio Bizzio. “Los nuevos". Página/12, 12 de abril de 1982, p. 12.

Harris, Wendell. "La canonicidad". El canon literario. Ed. Eric Sullà. Madrid, Arco/Libros, 1998, pp. 37-61. Impreso.

Horacio. Arte poética y otros poemas. Bogotá, Instituto Caro y Cuervo, 1974. Impreso.

Klein, Paula. "Un ejercicio de olvido: algunas líneas de lectura de Babel. Revista de Libros (1988-1991)". Revistas culturales, vol. 2, 2014, pp. 6-20.

Latour, Bruno. Reensamblar lo social: una introducción de la teoría del actor-red. Buenos Aires, Manantial, 2008. Impreso.

Lozares Colina, Carlos. "La teoría de redes sociales". Papers: revista de sociología, vol. 48, 1996, pp. 103-126.

Ludmer, Josefina. El cuerpo del delito. Un manual. Buenos Aires, Editorial Perfil, 1999. Impreso.

Maíz, Claudio. "Las re(d)vistas latinoamericanas y las tramas culturales: Redes de difusión en el romanticismo y el modernismo". Cuadernos del CILHA, vol. 12, no. 14, 2011, pp. 75-91.

"Tramas culturales. De las determinaciones sociales a la red intelectual". Anos 90, vol. 20, no. 37, 2013, pp. 19-35.

Mattoni, Silvio. "Diario de poesía: un reportaje universal". El matadero, vol. 9, 2015, pp. 47-54.

Merbilhaá, Margarita. "El estudio de las formas materiales de la sociabilidad intelectual: algunas cuestiones metodológicas en torno a las redes entre escritores latinoamericanos en Europa". VIII Congreso Internacional Orbis Tertius de Teoría y Crítica Literaria, 9, mayo, 2012, La Plata, Centro de Estudios de Teoría y Crítica Literaria, Facultad de Humanidades y Ciencias de la Educación, pp. 23-40.

Molina, Eugenia. “Aportes para un estudio del movimiento romántico argentino desde la perspectiva metodológica de redes (18301852)". Universum, vol. 15, 2000, pp. 399-43. 
Molina, José Luis. "La ciencia de las redes". Apuntes de Ciencia y Tecnología, vol. 11, 2004, pp. 36-42.

Moreno, María. "La generación del ochenta". Página/12, 28 de diciembre de 2003, http://www.paginal2.com.ar/diario/suplementos/radar/9-1149.html

Noguerol Jiménez, Francisca. "Narrar sin fronteras". Entre lo local y lo global: la narrativa latinoamericana en el cambio de siglo, 1990-2006. Eds. Jesús Montoya Juárez y Ángel Esteban, Madrid, Iberoamericana Editorial, 2008, pp. 19-35. Impreso. "Últimas tendencias y promociones". Historia de la Literatura Hispanoamericana, Tomo III, siglo XX. Ed. Trinidad Barrera, Madrid, Cátedra, 2008, pp. 167-179. Impreso.

Patiño, Roxana. "Revistas literarias y culturales argentinas de los 80 : usinas para pensar una época". Ínsula: revista de letras y ciencias humanas, vol. 715, 2006, pp. 2-5.

Pazos, Salvador. "Entrevista”. Babel. Revista de Libros, no. 6, 1989, p. 6.

Rama, Ángel. "La modernización literaria latinoamericana (18701910)". Hispamérica, vol. 36, 1983, pp. 3-19.

Ricagno, Alejandro. "Las combinaciones debidas". Babel. Revista de Libros, no. 14, 1990, p. 44.

Ringer, Fritz. "El campo intelectual, la historia intelectual y la sociología del conocimiento". Prismas. Revista de Historia Intelectual, no. 8, 2004, pp. 99-118.

Rodríguez Alfonso, Adriana. "Presencias objetuales: Walter Benjamin, montaje e imagen en la escritura reciente de Sergio Chejfec". Tonos digital: Revista de Estudios Filológicos, no 39, 2020, pp. $1-22$.

Said, Edward. Representations of the intellectual. Nueva York, Pantheon Books, 1994. Impreso.

Sarlo, Beatriz. "Intelectuales y revistas". América. Cahiers du CRICCAL, no. 10, 1992, pp. 9-16.

"La mesa de luz". Babel. Revista de Libros, no. 3, 1988, p. 20.

Warley, Jorge. "Los de treinta y pico. Abran cancha”. El Porteño, 1990, pp. 37-39.

Williams, Raymond. Problems in materialism and culture: Selected essays. Nueva York, Verso, 1980. Impreso. 
Sociology of culture. Chicago, Chicago University Press, 1982. Impreso.

Zanetti, Susana. "Modernidad y religación: una perspectiva continental (1880-1916)". América latina: Palabra, Literatura e Cultura, no. 2, 1994, pp. 489-534. 